\title{
Ibandronate - a convenient and effective treatment option for the management of post-menopausal osteoporosis
}

Julie Adkins

CSF Medical Communications, Cheltenham, UK

Drugs in Context 2012

DOI: http://dx.doi.org/10.7573/dic.212224

Citation: Adkins, J. Ibandronate - a convenient and effective treatment option for the management of post-menopausal osteoporosis. Drugs in Context: e212224. doi:10.7573/dic.212224

Copyright: this is an open access article published under the terms of the Creative Commons License Deed (CG BY-NG-ND 3.0) which allows you to share, copy, distribute and transmit the work provided it is properly attributed. You may not use this work for commercial purposes. For further information on commercial use, contact publisher@justmedicalmedia.com or go to wrerw.drugsincontext.com/copyright.

Search criteria: English language articles were identified by searching the PubMed database using the search terms 'ibandronate', 'ibandronic acid', 'Bonviva ${ }^{\mathbb{R}}$ ' and 'Bondronat ${ }^{\mathbb{R}}$. Abstracts were evaluated and selected for further review according to our standard protocols. Bibliographies of individual articles were also assessed for additional articles of interest and the manufacturer of ibandronate was contacted and invited to supply any additional data to that identified via the PubMed database.

Date of last literature search: 18 March 2008.

Conclusion: Intermittent oral and intravenous (IV) administration of the bisphosphonate ibandronate (150mg monthly orally; 3mg 3-monthly IV) provides an effective and convenient treatment option for the management of post-menopausal osteoporosis. The availability of these simplified oral and IV dosing regimens may improve patient adherence and ultimately clinical outcome.

This article was originally published by CSF Medical Communications Ltd (CSF) in Drugs in Context 2008;4(2):121-34. Drugs in Context and all CSF copyrights were acquired by Just Medical Media Ltd in 2009. 


\section{SUMMARY}

Osteoporosis is a progressive disease associated with significant morbidity and mortality, particularly in elderly post-menopausal women. Although the bisphosphonates as a class are considered by a number of leading authorities to be first-line therapy for the management of post-menopausal osteoporosis, some forms of bisphosphonate therapy require the administration of what for many may be inconvenient dosing regimens; this may have a negative impact on treatment adherence and potentially on long-term clinical outcome. The nitrogen-containing bisphosphonate, ibandronate (ibandronic acid), was marketed initially as a once-daily $(2.5 \mathrm{mg})$ oral formulation but is now also available as a single $150 \mathrm{mg}$ tablet for once-monthly administration and as an intravenous (IV) bolus injection (3mg/3ml) to be administered once every 3 months. The efficacy of daily administration of ibandronate $(2.5 \mathrm{mg})$ for the management of post-menopausal osteoporosis has been established. In clinical studies, intermittent oral and IV administration of ibandronate has been shown to be at least as effective as once-daily ibandronate in terms of improving lumbar spine and proximal femur bone mineral density (BMD) in post-menopausal women. Recent findings from a meta-analysis also suggest that these improvements in BMD with intermittent ibandronate may translate into significant risk reductions for non-vertebral and symptomatic vertebral fractures. The safety and tolerability profiles of the intermittent IV and oral ibandronate regimens appear to be similar to that of the once-daily ibandronate regimen (which has been compared to placebo in several studies in terms of its tolerability profile). Ibandronate has demonstrated good efficacy and tolerability in the management of post-menopausal osteoporosis and by virtue of its convenient intermittent oral and IV dosage schedule, represents a useful alternative to other currently available anti-osteoporotic medications.

Key words: Ibandronic acid, Bonviva ${ }^{\circledR}$, Bondronat ${ }^{\circledR}$, osteoporosis, bone resorption

\section{OSTEOPOROSIS: A PERSPECTIVE}

Osteoporosis is a progressive disease characterised by a reduction in bone mass and microarchitectural deterioration of the bone, resulting in decreased bone strength and increased risk of fracture, particularly of the hip, spine and distal forearm. ${ }^{1}$ An imbalance between osteoclast-mediated bone resorption and osteoblast-mediated bone formation is key to the development of osteoporosis. Osteoporotic fragility fractures occur as a result of mechanical forces that would not normally cause fracture and the majority of these fractures present clinically. However, vertebral fractures may be asymptomatic or remain undiagnosed in up to two thirds of patients and can lead to debilitating symptoms, with chronic back pain and spinal deformity. Patients in this situation also have a high risk of developing fractures elsewhere in the spine and at other sites. ${ }^{2}$
At present, approximately 75 million people are affected by osteoporosis in Europe, Japan and the USA; the estimated lifetime risk for wrist, hip and vertebral fractures is approximately $15 \%$ which is similar to that for coronary heart disease. ${ }^{3}$ Of all osteoporotic fractures, hip fractures have the most devastating consequences; at 1 year, hip fractures are associated with mortality in $20 \%$ of patients and $50 \%$ of patients are unable to live independently. ${ }^{1}$ Because of the aging population, the incidence of hip fractures is rising and is expected to increase 2- to 3-fold in the United States by 2040. ${ }^{4}$ Worldwide estimates have put the incidence of hip fractures at 6 million cases per year by 2050, a 3.5-fold increase from the 1.7 million cases reported in $1990 .^{5}$

The risk of fractures also increases exponentially with age, with the majority of individuals affected aged over 75 years. This age-related increase can be attributed to a 
gradual reduction in bone mass from the age of 40 years onwards which arises from an increase in osteoclast-mediated bone breakdown and a reduction in osteoblast-mediated bone formation. Although osteoporosis can develop in both men and women, it is particularly prevalent in post-menopausal women who have a reduction in oestrogen-mediated suppression of bone resorption leading to accelerated bone loss. ${ }^{6}$ The prevalence of osteoporosis in the UK has been put at approximately $21 \%$ for women aged between 50 and 84 years, with a lifetime risk of developing an osteoporotic fracture at age 50 years of $53 \%$. The corresponding figures for men are considerably lower at $6 \%$ and $21 \%$, respectively. ${ }^{7,8}$

The World Health Organization definition of osteoporosis relates bone mineral density (BMD) (as measured by dual energy x-ray absorptiometry [DEXA]) to the mean BMD of young adult women (T-score). Using this approach, a normal $\mathrm{T}$-score is defined as a BMD greater than 1.0 standard deviations (SD) below the reference mean (i.e. a T-score $\geq$-1) whereas osteoporosis is defined as a BMD at least 2.5 SDs below average (i.e. a T-score $\leq-2.5) .{ }^{9}$ However, although BMD score is a valuable diagnostic guide, it is only one of a number of clinical risk factors requiring consideration, as osteoporotic fractures can also occur in patients with BMD values above the defined level. ${ }^{10}$ Patients should therefore also be assessed for clinical risk factors that contribute to fracture risk independently of BMD; these include age, body mass index, previous fragility fracture, family history of fracture, use of oral glucocorticoid medication, smoking, alcohol intake, falls and coexistent rheumatoid arthritis. ${ }^{2}$

Osteoporotic fractures can have devastating consequences and are associated with marked functional impairment, poor quality of life, loss of independence and mortality. Because of the significant morbidity and mortality associated with osteoporosis, the disease has a major impact on public health and significant implications for healthcare expenditure. In the United States, the costs associated with osteoporotic fractures have been cited as comparable to those for cardiovascular disease and asthma. ${ }^{3}$ The annual direct costs for osteoporotic fractures have been estimated at approximately 32 billion Euros in Europe (2000 costs) and $\$ 12$ to $\$ 18$ billion in the United States (2002 costs). ${ }^{112}$ Further increases in costs to 77 billion Euros in 2050 have been estimated for Europe based on expected changes in demography. ${ }^{12}$

The goal of treatment for osteoporosis is to reduce fracture risk by slowing bone loss and improving bone mass and quality. Lifestyle measures are recommended to improve bone health and these include maintaining adequate dietary calcium intake (typically $1200 \mathrm{mg} /$ day for patients with osteoporosis) ${ }^{13}$ and normal vitamin D status, regular weight-bearing exercise, moderation of alcohol intake and cessation of smoking. ${ }^{3}$ Therapeutic options for osteoporosis include the bisphosphonates (ibandronate, alendronate and risedronate), raloxifene, strontium ranelate, calcitonin, calcium and vitamin D supplementation and parathyroid hormone peptide (teriparatide). Of these interventions, the bisphosphonates are considered a first-line therapy option. . $^{214,15}$

The bisphosphonates have a strong affinity for hydroxyapatite of the bone mineral matrix from where they are taken up by osteoclasts inhibiting osteoclast activity and bone resorption. ${ }^{16}$ Traditionally, the bisphosphonates have mainly been available for daily oral administration; however, the need for long-term administration coupled with adverse effects such as poor gastrointestinal tolerability often lead to poor adherence, with many patients discontinuing therapy. In an effort to improve the tolerability profile and adherence with these agents, intermittent bisphosphonate regimens have been evaluated. The bisphosphonate ibandronate (ibandronic acid) is available as an oral or intravenous (IV) formulation 
for convenient intermittent administration once monthly (oral) or 3-monthly (IV). The availability of an IV formulation also offers an alternative treatment option for those patients for whom oral administration may be inappropriate (e.g. patients with gastrointestinal [GI] intolerance) or for those with medication compliance problems because of cognitive impairment or co-prescription of several other medications.

\section{PHARMACOLOGY}

Ibandronate is a nitrogen-containing bisphosphonate with a tertiary nitrogen group on its R2 side chain and a hydroxyl group on its R 1 side chain. This structural confirmation confers ibandronate with a strong binding affinity for hydroxyapatite crystals in the mineral matrix of bone. Ibandronate inhibits osteoclast-mediated bone resorption and turnover through its affinity for hydroxyapatite but does not directly affect bone formation. In common with other bisphosphonates which bind to hydroxyapatite, ibandronate accumulates at sites of bone resorption and is then selectively internalised by bone-resorbing osteoclasts where it interferes with biochemical processes to decrease bone loss, increase BMD and decrease bone turnover. ${ }^{17}$ In terms of inhibition of bone resorption, ibandronate is 2-, 10- and 50-fold more potent than risedronate, alendronate and pamidronate, respectively, in the arotinoid-stimulated bone resorption rat model assay. ${ }^{18}$

Ibandronate also inhibits osteoclast activity through an inhibitory effect on intracellular pathways, namely the mevalonate pathway, via inhibition of farnesyl pyrophosphate synthase (FPPS). Disruption of this pathway at the level of FPPS, ultimately leads to a disruption in normal osteoclast morphology and function. ${ }^{19}$

\section{Pharmacokinetics}

After oral administration, ibandronate is rapidly absorbed in the upper GI tract $\left(\mathrm{t}_{\max }<1\right.$ hour) and has a low bioavailability $(0.63 \%)$. Coadministration of food or drink significantly reduces the bioavailability (by up to 90\%); however, bioavailability is not affected by ingestion of plain water. ${ }^{20,21}$ Therefore it is recommended that ibandronate is taken first thing in the morning, with a full glass of tap water, and that no food or drink or other medication is taken for at least 60 minutes.

After absorption, ibandronate either rapidly binds to the bone or is excreted unchanged in the urine. Ibandronate is not metabolised in humans and therefore does not inhibit the cytochrome $\mathrm{P} 450$ system and has a low potential for drug interactions. Approximately 40 to $50 \%$ of a circulating dose is removed from the circulation via the bone. ${ }^{21,22}$ Because ibandronate is sequestered in the bone, plasma elimination of the drug is multiphasic with a rapid and early decline in plasma concentration characterised by renal clearance and distribution into the bone; this is followed by a slower clearance phase as ibandronate is slowly released from the bone compartment into the blood. Plasma protein binding is moderate, exceeding 85\%. Total clearance of ibandronate is low (mean range 84 to $160 \mathrm{ml} / \mathrm{min})$. Renal clearance is related to creatinine clearance $(\mathrm{CrCl})$ and accounts for 50 to $60 \%$ of the total clearance. Ibandronate can be administered to patients with mild to moderate renal impairment $(\mathrm{CrCl} \geq 30 \mathrm{ml} / \mathrm{min})$ without dose adjustment; however, ibandronate is not recommended in patients with severe renal impairment $(\mathrm{CrCl}<30 \mathrm{ml} / \mathrm{min})$. Differences in the pharmacokinetic profile of ibandronate due to race were not evident in a study of Caucasian and Japanese patients. ${ }^{23}$ 


\section{Dosing}

Ibandronate is available as a $150 \mathrm{mg}$ film-coated tablet for once-monthly oral administration and as a $3 \mathrm{mg} / 3 \mathrm{ml}$ prefilled syringe for IV bolus administration over 15 to 30 seconds once every 3 months. ${ }^{21,22}$ To maximise absorption, it is recommended that oral ibandronate is taken after an overnight fast ( $\geq 6$ hours) and 1 hour before the first food and drink of the day (other than water). ${ }^{24}$ The $150 \mathrm{mg}$ ibandronate tablet should be swallowed whole in the upright position with a glass of plain water. Other oral medications, including supplemental calcium or vitamin D preparations, should not be taken until at least one hour after ibandronate administration. Concomitant calcium and vitamin D supplementation is recommended in patients in whom dietary intake is considered inadequate.

\section{CLINICAL EFFICACY}

Several randomised, double-blind studies have evaluated the efficacy of ibandronate, using a variety of oral and intravenous dosing regimens for the management of osteoporosis in postmenopausal women. ${ }^{24-38}$ For the purposes of this review, only those data relating to the currently licensed intermittent dosing regimens (i.e. oral ibandronate $150 \mathrm{mg}$ monthly and IV ibandronate 3mg 3-monthly) are discussed in detail here. Other data relating to alternative ibandronate dosing schedules are included where necessary to set the scene for the data on the recommended intermittent dosing regimens.

\section{Intermittent oral ibandronate}

Following confirmation that the optimal daily oral ibandronate dose was $2.5 \mathrm{mg},{ }^{26}$ the efficacy of intermittent ibandronate dosing was investigated in a double-blind, Phase II study of 240 women (aged 55-75 years) with post-menopausal osteoporosis (lumbar and/or femoral neck T-score <-2.5 SD). Patients were randomised to treatment with oral ibandronate $2.5 \mathrm{mg} /$ day, intermittent ibandronate $(20 \mathrm{mg}$ on alternate days for the first 24 days followed by a 9-week drug-free period) or placebo. Increases in lumbar spine BMD from baseline with intermittent ibandronate were significantly greater than with placebo at 1 year $(p<0.01)$ and equivalent to the daily ibandronate regimen at 2 years (5.64 and 5.54\%, respectively). ${ }^{26}$ Biochemical markers of bone turnover, including osteocalcin and C-terminal telopeptide (CTX), also decreased significantly from baseline in both ibandronate groups.

The positive results from this study led investigators to conduct the large, Phase III, double-blind, randomised BONE (oral iBandronate Osteoporosis vertebral fracture trial in North America and Europe) study. This trial compared daily oral ibandronate $(2.5 \mathrm{mg})$ with intermittent oral ibandronate $(20 \mathrm{mg}$ on alternate days for 12 doses every 3 months with $>2$-month dose-free interval) or placebo over a period of 3 years in 2946 women aged 55 to 80 years with post-menopausal osteoporosis (lumbar spine BMD T-score $\leq 2.0$ ). ${ }^{28,29}$ Both the intermittent and daily ibandronate regimens produced significant and similar increases in lumbar spine and hip BMD and suppression of bone turnover. Vertebral anti-fracture efficacy was significantly greater with both ibandronate regimens compared with placebo $(p \leq 0.0006)$, with the daily and intermittent regimens producing reductions in vertebral fracture risk of $62 \%$ and $50 \%$, respectively, after 3 years. The overall patient population was at a generally low risk of non-vertebral fractures and non-vertebral fracture risk was not reduced by ibandronate in the overall population. However, in a retrospective, post-hoc analysis of patients considered 'high risk' (femoral neck BMD T-score <-3.0), daily ibandronate significantly $(p=0.013)$ reduced the incidence of non-vertebral fractures by $69 \%$ relative to placebo. ${ }^{28}$ 
The results of the BONE study provided proof of concept that significant antifracture efficacy could be achieved with an intermittently administered oral ibandronate; however, the dosing regimen used in the study was impractical. As a consequence, two further studies were initiated, MOPS (Monthly Oral Pilot Study) and MOBILE (Monthly Oral iBandronate In LadiEs) to evaluate the efficacy of a more simplified intermittent oral ibandronate dosing regimen (once monthly).

\section{MOBILE study}

Results from MOPS, a 3-month, randomised, double-blind, placebo-controlled, pilot study in women aged 55-80 years, demonstrated that oral ibandronate $100 \mathrm{mg}(\mathrm{n}=26)$ or $150 \mathrm{mg}$ once monthly $(\mathrm{n}=36)$ is effective at suppressing bone turnover in post-menopausal women. ${ }^{32}$ Significant reductions in serum and urinary CTX from baseline were also reported with once-monthly ibandronate compared with placebo in this study.

The efficacy of the currently licensed oral intermittent ibandronate regimen of $150 \mathrm{mg}$ once monthly was confirmed further in the 2-year, randomised, double-blind, Phase III MOBILE study. ${ }^{31,33}$. This was a non-inferiority study comparing intermittent oral ibandronate $50+50 \mathrm{mg}$ (single doses given on two consecutive days), $100 \mathrm{mg}$ or $150 \mathrm{mg}$ administered once monthly with the proven daily oral ibandronate regimen (2.5mg daily) as the active comparator in women (aged 55-80 years) with post-menopausal osteoporosis. All of the 1609 randomised patients were at least 5 years since the menopause, had a mean lumbar spine BMD T-score of -2.5 to -5.0 and received daily supplemental calcium (500mg) and vitamin D (400IU). The primary efficacy endpoint was the percentage increase from baseline in lumbar spine BMD as measured by DEXA at 1 year.

After 1 year, lumbar spine BMD increased by $4.3,4.1$ and $4.9 \%$ in the monthly dosing groups
(50 +50mg, $100 \mathrm{mg}$ and $150 \mathrm{mg}$, respectively) compared with $3.9 \%$ in the $2.5 \mathrm{mg}$-daily group. ${ }^{32}$ All three monthly regimens were non-inferior to the daily regimen and the $150 \mathrm{mg}$ regimen was significantly superior to the daily regimen $(p=0.002)$. Increases in proximal femur BMD (total hip, femoral neck, trochanter) were also reported, with the largest increases again reported in the $150 \mathrm{mg}$-monthly group. Similar reductions in serum CTX were observed in all treatment arms from 3 months onwards and at 1 year the median relative decrease from baseline was $75.8 \%$ for ibandronate $150 \mathrm{mg}$ monthly compared with $67.3 \%$ for the $2.5 \mathrm{mg}$-daily regimen.

Analysis of 2-year data confirmed the findings of the first-year data that monthly ibandronate is at least as effective as daily administration for the treatment of osteoporosis. ${ }^{33}$ At 2 years, lumbar spine BMD increased by 5.0, and 6.6\% from baseline in the daily and $150 \mathrm{mg}$-oncemonthly groups, respectively. All three of the monthly regimens were non-inferior to the daily treatment and the $150 \mathrm{mg}$-monthly dose was significantly more effective than the daily regimen $(p<0.001)$. In addition, impressive increases in proximal femur BMD (total hip, trochanter and femoral neck) were reported for all treatment regimens with the most substantial increases occurring with the $150 \mathrm{mg}$-monthly regimen. The marked reductions in serum CTX evident across all treatment arms at 3 months were maintained throughout the study to 2 years (56 to $68 \%$ median decrease from baseline) with consistently greater reductions reported with ibandronate $150 \mathrm{mg}$ monthly $(68 \%)$.

\section{Comparison with alendronate}

One comparative study, MOTION (Monthly Oral Therapy with Ibandronate for Osteoporosis iNtervention), has been conducted to compare the efficacy of once-monthly oral ibandronate with that of the bisphosphonate alendronate 
administered intermittently in women with postmenopausal osteoporosis. ${ }^{34}$ This 12-month, double-blind study enrolled 1760 women (aged 55-84 years) with a mean lumbar spine (L2-L4) BMD T-score of $<-2.5$ and $\geq-5$.0. Patients were randomised to receive oral ibandronate $150 \mathrm{mg}$ once monthly or oral alendronate $70 \mathrm{mg}$ once weekly in addition to daily supplemental calcium (500mg) and vitamin D (400IU). After 1 year of treatment, mean changes in BMD from baseline with ibandronate and alendronate were 5.1 and $5.8 \%(95 \%$ CI for the difference, -1.13 , $-0.23)$, respectively, for the lumbar spine and 2.9 and $3.0 \%$ (95\% CI, $-0.38,0.18)$, respectively, for total hip. Similar increases in trochanter and femoral neck BMD were also reported with both treatments. These findings met the criteria for non-inferiority between treatments and confirmed that monthly ibandronate is clinically comparable to weekly alendronate in terms of BMD improvements at the lumbar spine and hip in women with post-menopausal osteoporosis.

\section{Intermittent intravenous ibandronate}

Only one study, the large Phase III, randomised, double-blind DIVA (Dosing IntraVenous Administration) trial, has evaluated IV ibandronate at the approved dosage of $3 \mathrm{mg}$ administered once every 3 months. ${ }^{39}$ Other randomised double-blind trials evaluated IV ibandronate regimens ( $\leq 2 \mathrm{mg} 3$-monthly) that were subsequently found to be suboptimal. ${ }^{35-38}$

In the 2-year non-inferiority DIVA study, 1395 women aged 55-80 years with osteoporosis (defined as lumbar spine BMD T-score <2.5 but $\geq-5.0$ ), who were at least 5 years postmenopausal, were randomised to receive oral ibandronate $2.5 \mathrm{mg}$ daily or one of two IV regimens of ibandronate: $2 \mathrm{mg}$ every 2 months or $3 \mathrm{mg}$ every 3 months. Daily calcium (500 mg/day) and vitamin D (400IU/ day) supplementation were also provided to all patients. The oral ibandronate dose of
$2.5 \mathrm{mg} /$ day was selected as a comparator because of its proven ability to reduce osteoporotic fracture risk. ${ }^{28}$ At 1 year, the change in mean lumbar spine BMD from baseline (primary endpoint) was $+5.1 \%,+4.8 \%$ and $+3.8 \%$ in the IV ibandronate 2 and $3 \mathrm{mg}$ and oral ibandronate $2.5 \mathrm{mg}$ groups, respectively. The greater increases in lumbar spine BMD in the two IV ibandronate groups meant that these regimens were both noninferior and statistically superior $(p<0.001)$ to oral ibandronate $2.5 \mathrm{mg}$ /day. Greater increases in proximal femur BMD were also reported with IV ibandronate (2 and 3mg) compared with oral ibandronate (total hip 2.6 and $2.4 \%$ vs $1.8 \%$, respectively; femoral neck 2.0 and $2.3 \%$ vs $1.6 \%$; trochanter 4.1 and $3.8 \%$ vs $3.0 \%$ ); these differences reached statistical significance $(p<0.05)$ except for comparison of 2-monthly IV ibandronate vs daily oral ibandronate for femoral neck BMD. Serum CTX levels were markedly decreased from baseline to a similar extent in all three treatment arms at both 6 and 12 months. ${ }^{39}$

Subsequent publication of the 2-year results from the DIVA study supported the findings at one year, with both IV regimens (2mg every 2 months and $3 \mathrm{mg}$ every 3 months) significantly superior to the oral regimen in terms of increase in lumbar BMD at 2 years (6.4 and $6.3 \%$ vs $4.8 \%$, respectively, $p<0.001)$. Greater increases in proximal femur BMD (total hip, femoral neck and trochanter) were also reported with the IV regimens at 2 years compared with placebo although the difference was not statistically significant at the femoral neck. The greater increases in BMD reported with the IV regimens were also reflected in a significantly higher proportion of patients achieving predefined increases in lumbar spine $(\geq 6 \%)$ or total hip $\mathrm{BMD}(\geq 3 \%)$ in this study (Figure 1). Clinically meaningful and similar reductions in serum CTX concentrations (53 to $60 \%$ ) were also reported in all 3 treatment arms. $^{40}$ 
Figure 1. Percentage of patients with lumbar spine and total hip bone mineral density (BMD) increases from baseline at or above predefined levels after 2 years of treatment with ibandronate in the DIVA study. ${ }^{40}$

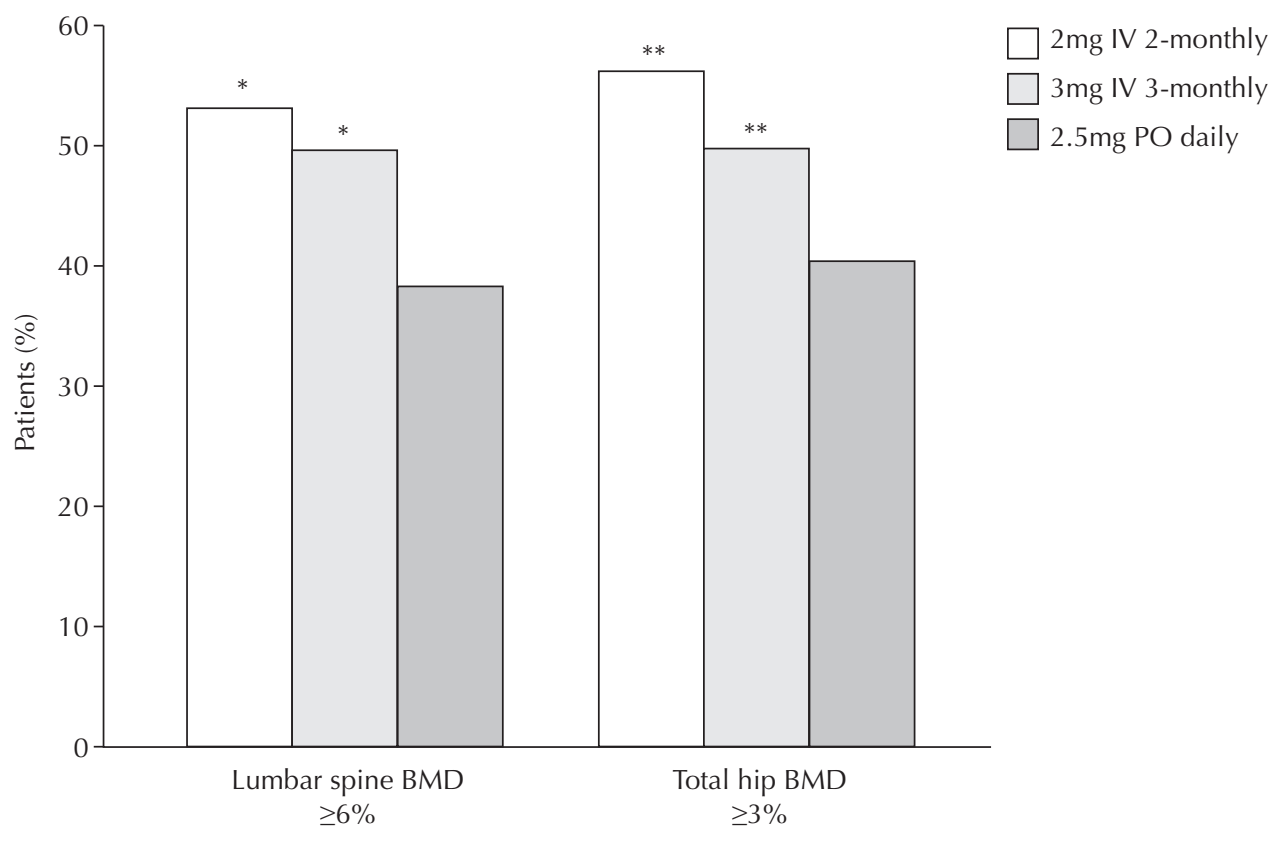

${ }^{*} p \leq 0.002,{ }^{* *} p \leq 0.014$ vs $2.5 \mathrm{mg}$ PO daily

\section{Fracture risk}

Reduction in fracture risk with intermittent oral and IV ibandronate has been assessed recently in a meta-analysis of data from the BONE, MOBILE, DIVA and IV fracture prevention studies. ${ }^{41}$ In this analysis, data were pooled from 8710 patients to determine whether higher ibandronate dose levels (annual cumulative exposure [ACE] $\geq 10.8 \mathrm{mg}$ ), which included the $150 \mathrm{mg}$-monthly oral regimen and the $3 \mathrm{mg} 3$-monthly IV regimen, were associated with reduced fracture risk compared with placebo. Six key non-vertebral fractures (clavicle, humerus, wrist, pelvis, hip and leg), all non-vertebral fractures, and clinical fractures, which included all non-vertebral fractures and symptomatic vertebral fractures, were analysed. The results of the meta-analysis suggested that oral ibandronate $150 \mathrm{mg}$ once monthly and IV ibandronate 3mg 3-monthly may provide significant non-vertebral fracture and clinical fracture efficacy; significant reductions in the adjusted relative risk of key non-vertebral fractures $(34.4 \% ; p=0.032)$, all non-vertebral fractures $(29.9 \% ; p=0.041)$ and clinical fractures $(28.8 \% ; p=0.010)$ were reported with high dose ibandronate (ACE $\geq 10.8 \mathrm{mg}$ ) compared with placebo. In addition, high-dose ibandronate was associated with a significantly $(p \leq 0.03)$ longer time to fracture versus placebo for all analysed fractures. ${ }^{41}$

\section{SAFETY AND TOLERABILITY}

Because the effective management of postmenopausal osteoporosis requires longterm therapy, it is important that drugs for osteoporosis are well tolerated. As a class, bisphosphonates have a generally favourable safety profile but may be associated with GI side effects, namely oesophagitis, oesophageal ulcers and dysphagia, particularly if the recommended dosing regimen is not followed (i.e. remaining 
upright for 30 to 60 minutes after oral administration). ${ }^{42,43}$ Other adverse events (AEs) commonly cited in the literature as associated with bisphosphonates include acute renal failure (primarily associated with zoledronic acid and clodronate) and influenza-like illness. Fatigue, fever, chills, myalgia and arthralgia are common symptoms of influenza-like illness, a condition that is usually transitory and selflimiting. Osteonecrosis of the jaw has also been associated with IV bisphosphonates. ${ }^{42,43}$ The incidence of all of these AEs varies considerably between the different bisphosphonates and also depends on the route and frequency of administration and dose.

\section{Intermittent oral ibandronate}

Safety data from clinical trials evaluating oral ibandronate administered continuously (2.5mg/day), weekly (20mg), or intermittently (20mg every other dose for 12 doses every 3 months) in women with post-menopausal osteoporosis indicate that ibandronate is well tolerated with an AE profile generally similar to that of placebo. ${ }^{24-28,30,31,38}$ In the largest of these studies, the 3-year BONE study, the incidence of treatment-related AEs was similar with ibandronate $2.5 \mathrm{mg}$ daily, intermittent ibandronate $20 \mathrm{mg}$ and placebo (19.8, 18.5 and $17.9 \%$, respectively) as was the incidence of serious treatment-related AEs $<1 \%$ in all 3 groups). ${ }^{28}$ GI AEs occurred with a similar incidence in the placebo and ibandronate groups (30 to $31 \%$ ), despite the fact that the study recruited a high proportion of patients with a history of GI disorders (approximately $30 \%$ of patients).

Two studies, MOPS and MOBILE, have evaluated the tolerability of intermittent oral ibandronate administered at the recommended dose of $150 \mathrm{mg}$ monthly. ${ }^{31,32}$ In the MOPS pilot study, oral ibandronate (150mg monthly) was reported to be well tolerated with an AE profile (including a GI tolerability profile) similar to that of placebo; the incidence of treatmentrelated AEs was 6 and $8 \%$, respectively. ${ }^{32}$ In the large Phase III MOBILE study ( $n=1609)$, the incidence and profile of AEs was similar with daily $(2.5 \mathrm{mg})$ and intermittent monthly administration of ibandronate $(50+50 \mathrm{mg}$, $100 \mathrm{mg}$ and $150 \mathrm{mg}$ ) over a 2-year treatment period. Drug-related AEs were reported in 30 to $37 \%$ of patients and only 8 treatment-related serious AEs were reported (gastric ulcer, $n=2$; duodenal ulcer, erosive duodenitis, gastric ulcer haemorrhage, gastritis haemorrhagic, melaena and liver disorder). ${ }^{33}$ Upper GI AEs occurred in 20 to $26 \%$ of patients and were generally mild to moderate in severity, and influenza-like illness (possibly/probably treatment related) was reported in 0.3 to $3.3 \%$ of patients. The flulike illness typically lasted 1 to 4 days and was associated with the first administered dose only.

Comparison of once-monthly oral ibandronate $150 \mathrm{mg}$ with weekly oral alendronate $70 \mathrm{mg}$ in the MOTION study revealed a similar incidence and profile of AEs in the two treatment groups. ${ }^{34}$ The incidence of musculoskeletal and general disorders (including influenza-like syndrome) was higher with ibandronate (6.8 vs 3.2\%). As in other studies, the flu-like symptoms caused by the acute-phase response generally occurred early in the course of treatment, were transient and did not require treatment.

\section{Intermittent intravenous ibandronate}

In common with data on the oral tolerability of ibandronate, safety data from placebo-controlled trials evaluating 3-monthly IV ibandronate at doses $\leq 2 \mathrm{mg}$, indicate that the drug is well tolerated, with a tolerability profile similar to that of IV placebo. ${ }^{34,37,38}$ In the large phase III DIVA study comparing IV ibandronate at the approved dose of $3 \mathrm{mg} 3$-monthly with ibandronate $2 \mathrm{mg}$ 2-monthly IV and 
$2.5 \mathrm{mg} /$ day orally, the overall incidence of AEs after one year with both IV regimens was similar to that with oral therapy (76 to $82 \%$ ). ${ }^{22,39}$ Treatment-related AEs were reported in $44 \%$ and $39 \%$ of patients in the 2-monthly and 3-monthly IV arms, respectively, and in $33 \%$ of patients in the daily oral arm. These most commonly included GI events (abdominal pain, dyspepsia, nausea), musculoskeletal events (arthralgia and myalgia) and influenza-like illness (Table 1). The incidence of influenza-like illness was higher in the IV ibandronate groups (5.1 and $4.9 \%$ in the 2 -monthly and 3 -monthly arms, respectively) compared with $1.1 \%$ in the $2.5 \mathrm{mg}$-daily group; it predominantly occurred at the time of the first IV ibandronate dose but was generally mild to moderate in nature and transient, requiring no treatment. The incidence of GI treatment-related AEs was slightly lower with IV ibandronate than with oral treatment (11.7 vs $14.3 \%$ overall) (Table 1). No cases of acute renal function or avascular necrosis of the jaw were reported with IV ibandronate in the DIVA study. At 2 years the profile and incidence of AEs was again similar in the 3 treatment groups. ${ }^{40}$

\section{MEDICATION PREFERENCE AND ADHERENCE}

Because the effective prevention and treatment of postmenopausal osteoporosis requires continued long-term adherence with medication, the ability to offer patients convenient and practical treatment regimens is crucial. Although proven to reduce the incidence of osteoporotic fracture risk, oral bisphosphonates have traditionally required frequent administration along with relatively strict dosing guidelines including fasting preand post-dose to maximise oral bioavailability and maintenance of an upright posture to reduce the incidence of upper GI disturbances. Taken together, these requirements may be too onerous and therefore unacceptable to many
Table 1. Incidence of the most common treatmentrelated adverse events (AEs) reported after one year of treatment in the DIVA study. ${ }^{22}$

\begin{tabular}{lcc}
$\begin{array}{l}\text { AEs, } \mathbf{n} \\
\text { (\% patients) }\end{array}$ & $\begin{array}{c}\text { Ibandronate } \\
\text { 3mg IV 3-monthly } \\
(\mathbf{n = 4 6 9 )}\end{array}$ & $\begin{array}{c}\text { Ibandronate PO } \\
\mathbf{2 . 5 m g} \text { daily } \\
\text { (n=465) }\end{array}$ \\
\hline $\begin{array}{l}\text { Gastrointestinal } \\
\text { Gastritis }\end{array}$ & $5(1.1)$ & $4(0.9)$ \\
Diarrhoea & $5(1.1)$ & $2(0.4)$ \\
Abdominal pain & $13(2.8)$ & $15(3.2)$ \\
Dyspepsia & $12(2.6)$ & $18(3.9)$ \\
Nausea & $8(1.7)$ & $12(2.6)$ \\
Constipation & $5(1.1)$ & $7(1.5)$ \\
Musculoskeletal disorders & & $2(0.4)$ \\
Musculoskeletal pain & $5(1.1)$ & $4(0.9)$ \\
Arthralgia & $11(2.3)$ & $4(0.9)$ \\
Myalgia & $8(1.7)$ & \\
Other & & $4(0.9)$ \\
Influenza-like illness & $22(4.7)$ & $2(0.4)$ \\
Fatigue & $5(1.1)$ & $3(0.6)$ \\
Headache & $5(1.1)$ & $3(0.6)$ \\
Rash & $4(0.9)$ &
\end{tabular}

IV, intravenous; PO, oral

patients, having a negative impact on treatment adherence and ultimately on long-term clinical outcome. There is considerable evidence to suggest that patients are more likely to be adherent with treatment if less frequent, more simplified treatment regimens are used; this was clearly illustrated with the introduction into clinical practice of weekly as opposed to daily bisphosphonate therapy. ${ }^{44,45}$ However, even with weekly bisphosphonate dosing, adherence remains suboptimal in more than one-half of patients. ${ }^{45}$

Data from two clinical studies of almost 700 patients have demonstrated a strong patient preference for a monthly versus a weekly oral bisphosphonate regimen. ${ }^{46,47}$ These two studies, BALTO I and II (Bonviva ALendronate Trial in Osteoporosis), were 6-month, randomised, multicentre, open-label, cross-over studies that evaluated patient preference for monthly oral ibandronate or weekly oral alendronate using patient surveys. In BALTO I, involving 342 post-menopausal women recruited in the 
US, a significantly higher proportion of patients $(66 \% ; \mathrm{p}<0.0001)$ had a preference for monthly oral ibandronate $(150 \mathrm{mg})$ compared with $27 \%$ for weekly alendronate $(70 \mathrm{mg})$; approximately $7 \%$ of patients stated no preference for either regimen. The most frequent reason given by patients for their preference was 'ease of following a treatment regimen for a long time'. ${ }^{46}$ Similarly, in BALTO II, which recruited patients from both Europe and the US $(n=321)$, of those patients expressing a preference (93.1\%), the majority $(70.6 \%)$ preferred the monthly ibandronate regimen to the weekly alendronate regimen $(p<0.0001) .{ }^{47}$

Although medication adherence may be improved if a patient has a preference for a particular treatment it may not always translate into prolonged adherence. However, data from the UK PERSIST (PERsistence Study of Ibandronate verSus alendronaTe) study suggest that less frequent dosing with monthly ibandronate may provide improved adherence. ${ }^{48}$ In this open-label study, 1054 post-menopausal women with osteoporosis (mean age 67.8 years) were randomised to once-monthly ibandronate (150mg) plus a patient support programme, or once-weekly alendronate $(70 \mathrm{mg})$ for 6 months. Persistence with treatment (primary endpoint) was significantly higher in the ibandronate compared with the alendronate group $(57 \%$ vs $39 \%$ of patients; $p<0.0001$ ), representing a $47 \%$ relative improvement with ibandronate. In addition, the proportions of patients remaining on treatment at study end or discontinuing from the study were also significantly different in favour of ibandronate plus patient support. However, it should be noted that the alendronate-treated patients in this study were not enrolled in a patient support programme.

The findings of the PERSIST study were in contrast to those from a study that used prescription-based data and online interviews to determine treatment preference and adherence among patients receiving weekly risedronate $(35 \mathrm{mg})$ or monthly ibandronate $(150 \mathrm{mg}) .{ }^{49}$
In this study, more patients preferred weekly risedronate over monthly ibandronate therapy (82\% vs 18\%, respectively, $p<0.0001)$; however, the prior information provided to patients about the fracture efficacy difference between the two treatments (in terms of the ability of risedronate to reduce both vertebral and a composite endpoint of non-vertebral fractures) may have biased this result. Persistence (144 vs 56 days; $p<0.0001)$ and adherence to therapy (73 vs 53\%; $p<0.0001$ ) were also reported to be significantly higher in the risedronate group over a 7-month period.

\section{DRUG INTERACTIONS}

Because ibandronate is not metabolised, the probability of interactions with other drugs is low. As mentioned previously, products such as antacids or vitamin formulations containing calcium or other multivalent cations (e.g. aluminium, magnesium and iron) can interfere with the absorption of ibandronate and should therefore be administered at least 6 hours before or 60 minutes after ibandronate. Since aspirin, non-steroidal antiinflammatory drugs (NSAIDs) and bisphosphonates are all associated with GI irritation, caution should be exercised when co-administering ibandronate with NSAIDs or aspirin.

Interference between bisphosphonates and bone-imaging agents is known to occur. However, specific studies evaluating ibandronate in this context have not been performed.

\section{COST-EFFECTIVENESS}

The significant morbidity and mortality associated with osteoporotic fractures results in significant healthcare costs to society. Few studies have been published on the cost-effectiveness of intermittent administration of bisphosphonates for the management of osteoporosis and no pharmacoeconomic studies have been identified that specifically evaluate ibandronate at the recommended IV and oral intermittent dosage regimens. 
Ibandronate was included in a sensitivity analysis in a recent study evaluating the costeffectiveness of alendronate $70 \mathrm{mg}$ weekly for the management of osteoporosis in postmenopausal women with clinical risk factors for fracture. ${ }^{50}$ The ibandronate analysis was modelled on data from a study evaluating oral ibandronate $2.5 \mathrm{mg}$ daily or an intermittent regimen of $20 \mathrm{mg}$ on alternate days for 12 doses every 3 months. ${ }^{28}$ Sensitivity analysis suggested that the cost-effectiveness of alendronate was much greater than that of ibandronate and other agents including strontium, raloxifene and risedronate (incremental cost-effectiveness ratios 9093-34,011 vs 867-3714 for alendronate).
Whether ibandronate in the recommended IV and oral intermittent dosage regimens will prove to be more cost-effective than other commonly used interventions has yet to be determined. However, the greater convenience of intermittent IV or oral ibandronate and associated improvements in patient adherence may have the potential to decrease the incidence of fractures and consequently reduce the consumption of healthcare resources. Specific cost-analyses of ibandronate will be required to confirm this hypothesis.

\section{KEY POINTS}

- Ibandronate is a potent nitrogen-containing bisphosphonate that inhibits osteoclast-mediated bone resorption in post-menopausal women.

- Although marketed originally as an oral formulation $(2.5 \mathrm{mg})$ for once-daily administration, ibandronate is now also available as a convenient $150 \mathrm{mg}$ tablet for once-monthly administration and as a $3 \mathrm{mg} / 3 \mathrm{ml}$ injection for quarterly intravenous administration.

- Intermittent oral and intravenous administration of ibandronate at recommended doses is at least as effective as once-daily ibandronate $(2.5 \mathrm{mg})$ in terms of improving lumbar spine and proximal femur bone mineral density in post-menopausal women.

- The improvements in bone mineral density reported with intermittent ibandronate may translate into significant risk reductions for non-vertebral and symptomatic vertebral fractures.

- Ibandronate, administered orally monthly or intravenously 3-monthly is at least as well tolerated as the daily regimen.

- Intermittent oral or intravenous ibandronate therapy may be particularly useful for patients with medication adherence problems. Intravenous ibandronate also offers an alternative treatment option for those patients for whom oral administration is contraindicated or poorly tolerated.

\section{REFERENCES}

1. Woolf AD, Pfleger B. Burden of major musculoskeletal conditions. Bull World Health Organ 2003; 81: 646-56.

2. Poole KE, Compston JE. Osteoporosis and its management. BMF 2006; 333: 1251-6.

3. Chan KM, Anderson M, Lau EM. Exercise interventions: defusing the world's osteoporosis time bomb. Bull World Health Organ 2003; 81: 827-30.

4. Schneider EL, Guralnik JM. The aging of America: Impact on health care costs. FAMA 1990; 263: 2335-40.
5. Cooper C, Campion G, Melton LJ. Hip fractures in the elderly: a world-wide projection. Osteoporos Int 1992; 2: 285-9.

6. Kanis JA, Pitt FA. Epidemiology of osteoporosis. Bone 1992; 13 (Suppl 1): S7-15.

7. van Staa TP, Dennison EM, Leufkens HG, Cooper C. Epidemiology of fractures in England and Wales. Bone 2001; 29: 517-22.

8. Kanis JA, Johnell O, Oden A et al. Risk of hip fracture according to the World Health Organization criteria for osteopenia and osteoporosis. Bone 2000; 27: 585-90. 
9. World Health Organization. Assessment of fracture risk and its application to screening for postmenopausal osteoporosis. Geneva:World Health Organization; 1994. Technical Report Series, No. 843.

10. Lane N. Osteoporosis: Is there a rational approach to fracture prevention? Bull NYU Hosp Ft Dis 2006; 64: $67-71$.

11. US Department of Health and Human Services. Bone Health and Osteoporosis: A Report of the Surgeon General. Washington, DC: US Department of Health and Human Services, Office of the Surgeon General, 2004. Available from: www.surgeongeneral.gov/library/bonehealth/content.html (Accessed 14 April 2008).

12. Kanis JA, Johnell O. Requirements for DXA for the management of osteoporosis in Europe. Osteoporos Int 2005; 16: 229-38.

13. National Osteoporosis Society. Information sheet on supplements of calcium and vitamin $\mathrm{D}$ with osteoporosis treatments. Available from:www.nos.org.uk/dr_media/ nos/Supplements_of_calcium_\&_vitamin_D_13-May-08. pdf (Accessed 12th May 2008).

14. National Institute for Clinical Excellence. Bisphosphonates (alendronate, etidronate, risedronate), selective oestrogen receptor modulators (raloxifene) and parathyroid hormone (teriparatide) for the secondary prevention of osteoporotic fragility fractures in postmenopausal women. Technology Appraisal 87 January 2005. Available from: www.nice.org.uk/ nicemedia/pdf/TA087guidance.pdf (Accessed 9th April 2008).

15. Cesareo R, Iozzino M, Alva D et al. Evidence based medicine and effective interventions of pharmacological therapy for the prevention of osteoporotic fractures. Minerva Endocrinol 2007; 32: 275-95.

16. Russell RG, Xia Z, Dunford JE et al. Bisphosphonates: an update on mechanisms of action and how these relate to clinical efficacy. Ann N Y Acad Sci 2007; 1117: 209-57.

17. Chapurlat RD. Clinical pharmacology of potent new bisphosphonates for postmenopausal osteoporosis. Treat Endocrinol 2005; 4: 115-25.

18. Muhlbauer RC, Bauss F, Schenk R et al. BM 21.0955, a potent new bisphosphonate to inhibit bone resorption. f Bone Miner Res 1991; 6: 1003-11.

19. Russell RG. Ibandronate: pharmacology and preclinical studies. Bone 2006; 38 (4 Suppl 1): S7-12.

20. Barrett J, Worth E, Bauss F, Epstein S. Ibandronate: a clinical pharmacological and pharmacokinetic update. 7 Clin Pharmacol 2004; 44: 951-65.

21. Roche Products Limited. Bonviva 150mg film-coated tablets: summary of product characteristics. 2006a. Available from www.emc.medicines.org.uk (Accessed April 16, 2008).

22. Roche Products Limited. Bonviva $3 \mathrm{mg} / 3 \mathrm{ml}$ solution for injection in pre-filled syringe: summary of product characteristics. 2006b. Available from www.emc.medicines. org.uk (Accessed April 16, 2008).

23. Pillai G, Gieschke R, Goggin T et al. Population pharmacokinetics of ibandronate in Caucasian and Japanese healthy males and postmenopausal females. Int $\mathcal{f}$ Clin Pharmacol Ther 2006; 44: 655-67.

24. Tankó LB, Felsenberg D, Czerwifski E et al. Oral weekly ibandronate prevents bone loss in postmenopausal women. f Intern Med 2003; 254: 159-67.
25. Ravn P, Glemmesen B, Riis BJ, Christiansen C. The effect on bone mass and bone markers of different doses of ibandronate: a new bisphosphonate for prevention and treatment of postmenopausal osteoporosis: a 1-year, randomized, double-blind, placebo-controlled dose-finding study. Bone 1996; 19: 527-33.

26. Riis BJ, Ise J, von Stein T, Bagger Y, Christiansen C. Ibandronate: a comparison of oral daily dosing versus intermittent dosing in postmenopausal osteoporosis. 7 Bone Miner Res 2001; 16: 1871-8.

27. Cooper C, Emkey RD, McDonald RH et al. Efficacy and safety of oral weekly ibandronate in the treatment of postmenopausal osteoporosis. 7 Clin Endocrinol Metab 2003; 88: 4609-15.

28. Chesnut III CH, Skag A, Christiansen C et al. Effects of oral ibandronate administered daily or intermittently on fracture risk in postmenopausal osteoporosis. 7 Bone Miner Res 2004; 19: 1241-9.

29. Delmas PD, Recker RR, Chesnut CH et al. Daily and intermittent oral ibandronate normalize bone turnover and provide significant reduction in vertebral fracture risk: results from the BONE study. Osteoporos Int 2004; 15: 792-8.

30. McClung MR, Wasnich RD, Recker $\mathrm{R}$ et al. Oral daily ibandronate prevents bone loss in early postmenopausal women without osteoporosis. 7 Bone Miner Res 2004; 19: 11-8.

31. Miller PD, McClung MR, Macovei L et al. Monthly oral ibandronate therapy in postmenopausal osteoporosis: 1-year results from the MOBILE study. 7 Bone Miner Res 2005; 20: 1315-22.

32. Reginster JY, Wilson KM, Dumont E, Bonvoisin B, Barrett J. Monthly oral ibandronate is well tolerated and efficacious in postmenopausal women: results from the monthly oral pilot study. 7 Clin Endocrinol Metab 2005; 90: 5018-24.

33. Reginster JY, Adami S, Lakatos $\mathrm{P}$ et al. Efficacy and tolerability of once-monthly oral ibandronate in postmenopausal osteoporosis: 2 year results from the MOBILE study. Ann Rheum Dis 2006; 65: 654-61.

34. Miller PD, Epstein S, Sedarati F, Reginster JY. Oncemonthly oral ibandronate compared with weekly oral alendronate in postmenopausal osteoporosis: results from the head-to-head MOTION study. Curr Med Res Opin 2008; 24: 207-13.

35. Thiébaud D, Burckhardt P, Kriegbaum H et al. Three monthly intravenous injections of ibandronate in the treatment of postmenopausal osteoporosis. Am $\mathcal{F}$ Med 1997; 103: 298-307.

36. Stakkestad JA, Benevolenskaya LI, Stepan JJ et al. Intravenous ibandronate injections given every three months: a new treatment option to prevent bone loss in postmenopausal women. Ann Rheum Dis. 2003; 62: 969-75.

37. Adami S, Felsenberg D, Christiansen C et al. Efficacy and safety of ibandronate given by intravenous injection once every 3 months. Bone 2004; 34: 881-9.

38. Recker R, Stakkestad JA, Chesnut CH et al. Insufficiently dosed intravenous ibandronate injections are associated with suboptimal antifracture efficacy in postmenopausal osteoporosis. Bone 2004; 34: 890-9. 
39. Delmas PD, Adami S, Strugala G et al. Intravenous ibandronate injections in postmenopausal women with osteoporosis: one-year results from the dosing intravenous administration study. Arthritis Rheum 2006; 54: 1838-46.

40. Eisman JA, Civitelli R, Adami S et al. Efficacy and tolerability of intravenous ibandronate injections in postmenopausal osteoporosis: 2-year results from the DIVA study. F Rheumatol 2008; 35: 488-97.

41. Harris ST, Blumentals WA, Miller PD. Ibandronate and the risk of non-vertebral and clinical fractures in women with postmenopausal osteoporosis: results of a meta-analysis of phase III studies. Curr Med Res Opin 2008; 24: 237-45.

42. Diel IJ, Bergner R, Grötz KA. Adverse effects of bisphosphonates: current issues. 7 Support Oncol 2007; 5: 475-82.

43. Strampel W, Emkey R, Civitelli R. Safety considerations with bisphosphonates for the treatment of osteoporosis. Drug Saf 2007; 30: 755-63.

44. Cramer JA, Amonkar MM, Hebborn A, Altman R. Compliance and persistence with bisphosphonate dosing regimens among women with postmenopausal osteoporosis. Curr Med Res Opin 2005; 21: 1453-60.

45. Recker RR, Gallagher R, MacCosbe PE. Effect of dosing frequency on bisphosphonate medication adherence in a large longitudinal cohort of women. Mayo Clin Proc 2005; 80: 856-61.
46. Emkey R, Koltun W, Beusterien K et al. Patient preference for once-monthly ibandronate versus once-weekly alendronate in a randomized, open-label, cross-over trial: the Boniva Alendronate Trial in Osteoporosis (BALTO). Curr Med Res Opin 2005; 21: 1895-903.

47. Hadji P, Benhamou C-L, Devas V, Masanauskaite D, Barrett-Connor E. Women with postmenopausal osteoporosis prefer once-monthly oral ibandronate to weekly oral alendronate: results of BALTO II. Osteoporos Int 2006, 17 (Suppl 1): S69.

48. Cooper A, Drake J, Brankin E; the PERSIST Investigators. Treatment persistence with once-monthly ibandronate and patient support $v s$. once-weekly alendronate: results from the PERSIST study. Int 7 Clin Pract 2006; 60: 896-905.

49. Gold DT, Safi W, Trinh H. Patient preference and adherence: comparative US studies between two bisphosphonates, weekly risedronate and monthly ibandronate. Curr Med Res Opin 2006; 22: 2383-91.

50. Kanis JA, Adams J, Borgström F et al. The cost-effectiveness of alendronate in the management of osteoporosis. Bone 2008; 42: 4-15.

Correspondence:

Stephen I'Anson, Just Medical Media Ltd, 11 Redgrove Park, Cheltenham, Gloucestershire, GL51 6QY, UK.

Email: publisher@justmedicalmedia.com. 